Proceedings of the 2004 lEEE

International Conference on Networking, Sensing \& Control

Taipei, Taiwan, March 21-23, 2004

\title{
Sliding Mode Control Reduced Chattering For Systems With Dependent Uncertainties
}

\author{
M. S. Chen \\ Department of Mechanical \\ Engineering, National Taiwan \\ University, Taipei 106, Taiwan \\ mschen@ccms.ntu.edu.tw
}

\author{
Y. R. Hwang \\ Department of Mechanical \\ Engineering, National Central \\ University, Chung-Li, Taiwan \\ yhwang@cc.ncu.edu.tw
}

\author{
M. Tomizuka \\ Department of Mechanical \\ Engineering, UC Berkeley \\ Berkeley, USA \\ tomizuka@me.berkeley.edu
}

\begin{abstract}
Previously, a paper is suggested to reduce the chattering of sliding mode control by on-line adjusting the width of the boundary layer based on the state norm of an uncertain linear system. It is claimed that the new design can effectively eliminate chattering while at the same time ensuring almost perfect control accuracy. In this note, simulations are presented to show that the state-dependent boundary layer design is effective only if the system is subject to state-dependent uncertainties and no external disturbance.
\end{abstract}

Keywords : variable structure system, sliding mode control, boundary layer control, chattering, control accuracy.

\section{Introduction}

Sliding mode control is known to be robust against parameter uncertainties and external disturbances $[1,2,3]$. However, for the sliding surface to be attractive, a switching function must be used in the control law, which causes chattering of the control signals. In order to reduce chattering, one can introduce a boundary layer $[4,5]$ around the sliding surface. Inside the boundary layer, the discontinuous switching function is interpolated by a continuous function to avoid discontinuity of the control signals. The width of the boundary layer is normally constant, and the larger the boundary layer width, the smoother the control signal. Even though the boundary layer design alleviates the chattering phenomenon, it no longer drives the system state to the origin, but to a small residual set around the origin. The size of the residual set is determined by the width of the boundary layer: the larger the width of the boundary layer, the larger the size of the residual set. As a consequence, there exists a design conflict between requirements on the smoothness of control signals and on the control accuracy. For smoothness of the control signals, a large boundary layer width is preferred but for better control accuracy, a small boundary layer width is preferred.

For a review of the various designs that aim to reduce chattering of sliding mode control, one can refer to [6]. In Particular the authors in [6] propose scheduling the boundary layer width based on the system state; forming a "closed-loop" tuning of the boundary layer width. It is shown that under certain design parameter considerations, the closed-loop system can still be exponentially stable with this state-dependent boundary layer design control. However, it is recently found that the simulation results in [6] are not correct because of the wrong use of integration algorithm. In [6], the authors use the Matlab ODE45 (ODE solver) to simulate the continuous plant and the controller, for which the integration time step is variable (self-adjusted). Unfortunately, this self-adjusted time step scheme led us to an incorrect observation that the control chattering gradually disappears when the state approaches the origin along the sliding surface. In this note, we re-run the numerical examples, simulating more realistic situations : digital implementation of the sliding mode control for the continuous plant, in which the continuous plant dynamics is simulated with a $4^{\text {th }}$ Runge-Kutta scheme with a fix $3 \mathrm{ed}$ integrated time step $\Delta t=0.001$ second, and the sampling time for digitally implementing the sliding mode control is $T=0.05$ second. With the new simulations, it is found that the control chattering does not disappear as the state approaches the origin when there is persistent external disturbance. The chattering will gradually disappear as the state approaches the origin only when the system has state-dependent uncertainties but void of external disturbances. As a result of this finding, we need to modify our claim, although all proofs in [6] remain correct, as follows: The state-dependent boundary layer design proposed in [6] is able to eliminate the control chattering while ensuring almost perfect control accuracy only under the condition that there are state-dependent system uncertainties but void of external disturbances.

The remainder of this paper is organized as follows. Section 2 reviews the switching sliding mode control for linear uncertain systems. Section 3 examines the constant-width and decaying-width boundary layer controls. Section 4 introduces the state-dependent boundary layer control in [6], and presents the new simulation results. Section 5 gives the conclusions. All the proofs of lemmas and theorems can be found in [6]. 


\section{Switching Sliding Mode Control}

Consider a switching sliding mode control design for a linear system with "matching" uncertainties [7],

$$
\dot{x}=A x+B(u+\Delta E x+d), \quad x(0)=x_{0},
$$

where $x \in R^{n}$ is the system state, $u$ is a scalar control input, $A \in R^{n \times n}$ and $B \in R^{n}$ are nominal system matrices satisfying the controllability condition [8], uncertainty $\Delta E$ is possibly time-varying, and $d$ an unknown disturbance. The system uncertainties are bounded by two known constants :

$$
\|\Delta E\| \leq \bar{E}, \quad\|d\| \leq \bar{D}
$$

Note that one can always perform a state transformation such that the controllable pair

$(A, B)$ is in the controller canonical form [8]:

$$
A=\left[\begin{array}{cccc}
0 & 1 & . & . \\
\cdot & 0 & 1 & \cdot \\
\cdot & . & . & . \\
a_{1} & . & . & a_{n}
\end{array}\right], B=\left[\begin{array}{l}
0 \\
\cdot \\
0 \\
1
\end{array}\right] .
$$

The objective of sliding mode control is to regulate the state $x$ in (1) to zero, and this is achieved by a two-stage control design.

Stage I : design of the sliding variable. First define an augmented state

$$
\dot{v}=x_{1}, \text { or } v=\int_{0}^{t} x_{1} d \tau
$$

and choose the sliding variable as

$$
\begin{aligned}
& s=C x+c_{0} v, C=\left[c_{1}, c_{2}, c_{3}, \ldots . ., 1\right], \\
& =x_{n}+c_{n-1} x_{n-1}+\ldots . .+c_{1} x_{1}+c_{0} \int_{0} x_{1} d \tau, \\
& =x_{1}^{(n-1)}+c_{n-1} x_{1}^{(n-2)}+\ldots . .+c_{1} x_{1}+c_{0} \int_{0}^{r} x_{1} d \tau .
\end{aligned}
$$

where the coefficients $c_{i}^{\prime} s$ are chosen such that the differential equation (6) is stable (has only left-half plane characteristic roots). The purpose of adding an integral term in (6) is for the special case when the system dimension $\mathrm{n}=1$. Note from (3) and (5) that $C B=1$.

The differential equations (4) and (6) can be cast into a state space form :

$$
\dot{z}=F z+G s \text {, where } z=\left[\begin{array}{c}
\int_{0} x_{1} d \tau \\
x_{1} \\
\cdot \\
\cdot \\
x_{n-1}
\end{array}\right] \in R^{n},
$$

and matrices $F$ and $G$ are in controller canonical form :

$$
F=\left[\begin{array}{cccc}
0 & 1 & \cdot & \cdot \\
\cdot & 0 & 1 & \cdot \\
\cdot & \cdot & \cdot & \cdot \\
-c_{0} & \cdot & \cdot & -c_{n-1}
\end{array}\right] \in R^{n \times n}, G=\left[\begin{array}{l}
0 \\
\cdot \\
0 \\
1
\end{array}\right] \in R^{n}
$$

Since the differential equation (6) is stable by the choice of the coefficients $c_{i} s$, the matrix $F$ in (7) is stable.

Several results regarding (7) that will be repeatedly used in later sections are listed below. Firstly, given the stable matrix $F$ in (8), there exist positive constants $m$ and $\alpha$ such that

$$
\left\|e^{F(t-\tau)}\right\| \leq m e^{-\alpha(t-\tau)}, \forall t \geq \tau,
$$

where $\alpha$ is treated as a control design parameter since its value is determined by the choices of $c_{i} s$ in (6). Secondly, given any positive constant $\sigma \geq-\operatorname{Re}\left[\lambda_{i}(F)\right]>0$, where $\operatorname{Re}\left[\lambda_{i}(F)\right]$ denotes the real part of eigenvalues of $F$, there exists a positive definite matrix $P \in R^{n \times n}$ satisfying the following Lyapunov inequality:

$$
\begin{gathered}
(-F-\sigma I)^{T} P+P(-F-\sigma I) \leq 0, \\
\sigma \geq-\operatorname{Re}\left[\lambda_{i}(F)\right]>0, \forall i .
\end{gathered}
$$

Finally, from linear system theory [8], the state in (7) satisfies

$$
z(t)=e^{F t} z(0)+\int_{0} e^{F(t-\tau)} G s(\tau) d \tau .
$$

Stage II : design of the control input. The stable state space equation (7) suggests that if the sliding variable $s$ can be driven to zero by some control design, the state $z$ will decay to zero. Therefore, one chooses the following "switching" sliding mode control to drive $s$ to zero:

$$
u=-\sigma s-c_{0} x_{1}-C A x-\rho(x) f_{0}(s),
$$

Where $\sigma>0, s$ is the sliding variable $\rho(x)=\rho_{0}(\bar{E}\|x\|+\bar{D}), \rho_{0}>1$, with $\bar{E}$ and $\bar{D}$ given by $(2)$, and $f_{0}(s)$ a switching function:

$$
f_{0}(s)=\operatorname{sgn}(s)=\left\{\begin{array}{c}
1, s>0 \\
-1, s<0
\end{array}\right.
$$

Lemma 1 [3]: If the switching sliding mode control (12) is applied to the uncertain system (1), there exists a finite time To such that $|s(t)|=0$ for all $t>T_{0}$.

Once $s(t)$ becomes zero according to Lemma 1, the state $z$ in (7) starts to decay exponentially, and so does the state $x$ in (1).

Theorem 1 [3]: If the switching sliding mode control (12) is applied to the uncertain system (1), the system state $x$ will converge to zero exponentially. 


\section{Constant/Decaying-Width Boundary Layer Control}

In practical implementation of the switching control (12), the imperfect switching of the discontinuous function $f_{0}(s)=\operatorname{sgn}(s)$ causes the control signal to chatter [3]. Such chattering may bring damage to the actuator or excite high-frequency unmodelled dynamics. To alleviate the problem, a boundary layer around the sliding surface $s=0$ is suggested to smooth the control signal. The result is the so-called boundary layer control:

$$
u=-\sigma s-c_{0} x_{1}-C A x-\rho(x) f_{1}(s)
$$

where the discontinuous function $f_{0}(s)=\operatorname{sgn}(s)$ in the switching control (12) is now replaced by a continuous function

$$
f_{1}(s)=\frac{s}{|s|+\epsilon_{0} e^{-\pi t}}, \sigma>\pi \geq 0, \epsilon_{0}>0
$$

in which $\in_{0} e^{-\pi t}$ is the width of the boundary layer, which decays exponentially to zero when $\pi \neq 0$, and remains constant when $\pi=0$.

Lemma 2 : If the boundary layer control (14) is applied to the system (1), then

$$
|s(t)| \leq \eta_{0} e^{-\pi t}+|s(0)| e^{-\sigma t}, \eta_{0}=\frac{\epsilon_{0}}{\rho_{0}-1}
$$

If $\pi$ chosen to be positive, it is a decaying-width boundary layer design. In this case, exponential stability of the controlled system is guaranteed by the control (14).

Theorem 2: If the decaying-width boundary layer control (14) $(\pi>0)$ is applied to the uncertain system (1), the system state $x$ will converge to zero exponentially.

If $\pi$ is chosen to be zero, it is a constant-width boundary layer design. In this case, the so-called practical stability is guaranteed in the sense that given any small neighborhood of the origin, there always exist control design parameters in (14) such that the state will eventually be trapped in the specified neighborhood.

Theorem 3: If the constant-width boundary layer control (14) $(\pi=0)$ is applied to theuncertain system (1), the system state $\mathrm{x}$ will asymptotically approach a residual set aroundthe origin, with the size of the residual set proportional to $\epsilon_{0} /\left(\rho_{0}-1\right), \rho_{0}>1$.

\section{State-Dependent Boundary Layer Control}

In [6], a new boundary layer design is proposed for the sliding mode control, in which the width of the boundary layer is proportional to the state norm $\|x\|$. Hence, it is called a state-dependent boundary layer control:

$$
\begin{aligned}
u= & -\sigma s-c_{0} x_{1}-C A x-\rho(x) f_{2}(s) \\
& +\eta_{1}^{2} G^{T} P z+\eta_{\delta} \eta_{1} G^{T} P e_{z},
\end{aligned}
$$

where Pis as in (10), G as in (8), $z$ the state in(7),

$$
\begin{aligned}
& \eta_{1}=\epsilon_{1} /\left(\rho_{0}-1\right)>0, \eta_{0}=\epsilon_{0} /\left(\rho_{0}-1\right)>0, \\
& e_{z}=z /\|z\|_{p} \text { and } \\
& f_{2}(s)=\frac{s}{|s|+\varepsilon_{1}\|z\|_{p}+\varepsilon_{0}}, \\
& \|z\|_{p} \stackrel{\Delta}{=} \sqrt{z^{T} P z}
\end{aligned}
$$

in which $\epsilon_{1}>0$ and 1 . Since $\epsilon_{0} \approx 0$, the boundary layer width in (18) is approximately proportional to the state norm $\|z\|_{p}$. The reason for adding this small $\epsilon_{0}$ in the boundary layer width is to prevent $f_{2}(\cdot)$ from degenerating into the discontinuous sign (.) function when the state $z$ has decayed practically to zero. Note that there are two extra feedback terms (the last two terms) in the new control (17), which serve to ensure that the inequality (19) in Lemma 3 below holds.

Lemma 3 : If the new boundary layer control (17) is applied to the uncertain system (1), the sliding variable $s$ will be bounded by, for all $t>0$,

$$
\begin{aligned}
& |s(t)| \leq \eta_{1}\|z(t)\|_{p}+\eta_{0}+|s(0)| e^{-\sigma t}, \\
& \eta_{1}=\epsilon_{0} /\left(\rho_{0}-1\right), \eta_{0}=\varepsilon_{0} /\left(\rho_{0}-1\right)
\end{aligned}
$$

Lemma 4 (Bellman-Gronwall's Lemma) [18]: If a continuous function $f(t) \geq 0$ satisfies

$$
f(t) \leq b(t)+\int_{0} k(\tau) f(\tau) d \tau, \forall t \geq t_{0}
$$

where and are continuous and non-negative for all $t>$ to, then

$$
f(t) \leq b(t)+\int_{t_{0}} b(\tau) k(\tau) e^{\int_{\tau}^{t} k(v) d v} d \tau, \forall t \geq t_{0}
$$

Substituting the result of Lemma 3 into (II), and using the above Bellman-Gronwall's Lemma, one can establish the following stability result for the state-dependent boundary layer control.

Theorem 4: Consider the uncertain system (1) and the state-dependent boundary layer control (17). If the control design parameters are chosen to satisfy

$$
\begin{gathered}
\epsilon_{1}>0,1 \square \epsilon_{0}>0, \rho_{0}>1, \sigma \geq-\operatorname{Re}\left[\lambda_{i}(F)\right]>0 \\
, \alpha>\frac{\epsilon_{1}}{\rho_{0}-1} m \sqrt{\bar{\sigma}_{p}}
\end{gathered}
$$


where a and $m$ are as in (9), $\bar{\sigma}_{p}$ is the maximum singular value of $P$ in (10), $F$ is given by (8), then the system state $x$ will asymptotically approach a residual set around the origin, with the size of the residual set proportional to $\varepsilon_{0}$.

As mentioned in the Introduction, the variable integration time step used for solving the controlled-plant dynamics in [6] is not correct. Hence, in the following simulation examples, the system dynamics is simulated with a $4^{\text {th }}$ Runge-Kutta scheme with a fixed integration time step $\Delta t=0.001$ second, and the sampling time for digitally implementing the sliding mode control is $T=$ 0.05 second.

Example I : state-dependent boundary layer width with no disturbance

Consider an open-loop unstable system with uncertainties

$$
\begin{aligned}
& \dot{x}_{1}=x_{2} \\
& \dot{x}_{2}=u+\Delta E x_{2}
\end{aligned}
$$

where the parametric uncertainty $\Delta E=3$. Figure 1 shows the simulation results of the digital implementation of a constant width boundary layer $\operatorname{design}(15)$ in $[1]$ with $\varepsilon_{0}=0.001, \pi=0, \bar{E}=5$, $\bar{D}=0 . \rho_{0}=1, \sigma=2$, and $c_{0}=1, c_{1}=2, c_{2}=1$ in (6). It is seen from Figure 1 that chattering does show up because of the large sampling time (0.05 second), and the chattering amplitude dwindles as the state approaches the origin. To remove this chattering, one has to increase the boundary layer width up to a value $\varepsilon_{0}=0.5$. However, a large value of $\varepsilon_{0}$ results in a large steady state error of $\|x(t)\|$ as shown by Figure 2 , where $\lim _{t \rightarrow \infty}\|x(t)\| \approx 0.34$. The third simulation shows the digital implementation (still a 0.5 second sampling time) of the proposed state-dependent boundary layer design (18) with $\varepsilon_{0}=0.001, \varepsilon_{1}=0.4$. All the other design parameters and simulation conditions are the same as the contant-width boundary layer design. The results are plotted in Figure 3, which clearly shows that our proposed design has successfully eliminated the chattering in this case, and most importantly the system state approaches almost zero exponentially.

Example 2 : - with persistent external disturbance

Cinsider another system

$$
\begin{aligned}
& \dot{x}_{1}=x_{2} \\
& \dot{x}_{2}=u+d
\end{aligned}
$$

where the disturbance $d(t)=\sin (t)$. In the mistaken simulation ( a variable integration time step) of the constant width boundary layer control (15) in [6] ( $\varepsilon_{0}=0.001$ ), the control chattering gradually disappears as the state approaches the origin, as is shown in the upper plot of Figure 2 in [6]. Here, we re-run the simulation with a fixed integration time step as mentioned above. Figure 4 depicts the new simulation results of the digital implementation of the constant width boundary layer control (15) with all control parameters the same as in [6]. It is seen that under the correct simulation condition, the control chattering persistently exists in the case of a non-vanishing disturbance. Figure 5 then show the new simulation results of the state dependent boundary layer design (18) with $\varepsilon_{0}=0.001, \varepsilon_{1}=0.4$. Comparison of Figures 5 and 4 shows that the state dependent boundary layer design cannot remove chattering effectively in the case of a non-vanishing disturbance.

\section{Conclusions}

The new simulation results in this note indicate that removing the control chattering and acquiring almost perfect control accuracy at the same time is achievable for the sliding mode control when the system is subject to state-dependent uncertainties only. However, this goal becomes much more difficult when the system is subject to persistent external disturbances.Further research is still required to solve this dilemma.

\section{References}

[1] V. 1. Utkin, "Variable structure systems with sliding modes," IEEE Trans. Autom. Control, vol. AC-22, pp. 212-222, 1977.

[2] J. Y. Hung, W. B. Gao, and J. C. Hung, "Variable structure control: a survey," IEEI Trans. Ind Electron., vol. 40, pp. 2-22, 1993.

[3] J. J. E. Slotine and W. Li, Applied Nonlinear Control, Prentice-Hall, Englewood Cliffs, New Jersey, 1991.

[4] J. J. E. Slotine and S. S. Sastry, "Tracking control of nonlinear systems using sliding surfaces with application to robot manipulator," Int. J. Contr. vol. 38, no. 2, pp. 931-938,1983.

[5] J. A. Burton and A. S. 1. Zinober, "Continuous approximation of variable structure control," Int. $J$. System Science, vol. 17, no. 6, pp. 875-885, 1986.

[6] M. S. Chen, Y. r. Hwang, and M. Tomizuka, "A state-dependent boundary layer design for sliding mode control," IEEE Trans. Autom. Control, vol. AC-47, pp. 1677-1681, 2002.

[7] B. Drazenovic, "The invariance condition in variable structure systems", Automatica, vol.5, no.3, pp. 287-295, 1969.

[8] F. Callier, and C. A. Desoer, Linear System Theory, Springer-Verlag, New York, 1991.

[9] M. J. Corless, and G. Leitmann, "Continuous state feedback guaranteeing uniform ultimate boundedness for 
uncertain dynamic systems" IEEE Trans. Autom. Control, vol. AC-26; pp. 1139-1143, 1981.

[10] S. Sastry, Nonlinear Systems, Analysis, Stability, and Control, Springer-Verlag, New York, 1999

\section{Figures}
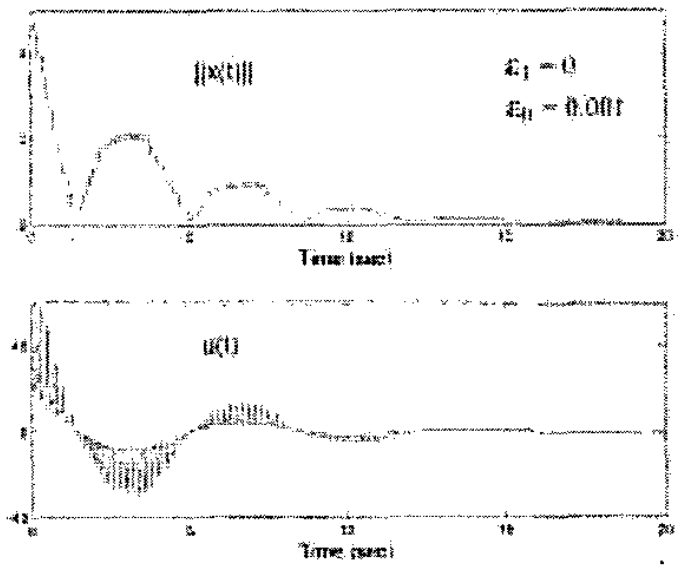

Figure 1. small boundary layer width design $\left(\varepsilon_{0}=0.001\right)$

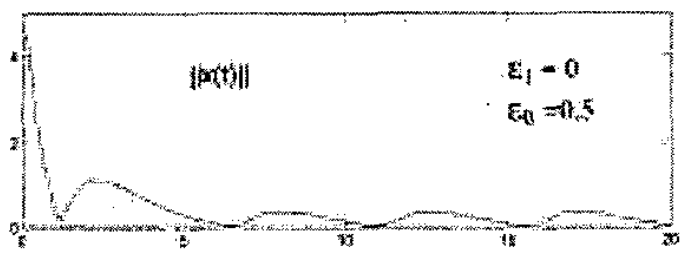

Trand tarit.

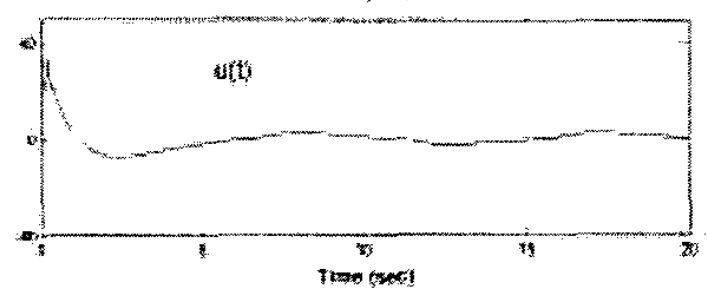

Figure 2. Large boundary layer width design $\left(\varepsilon_{0}=0.5\right)$
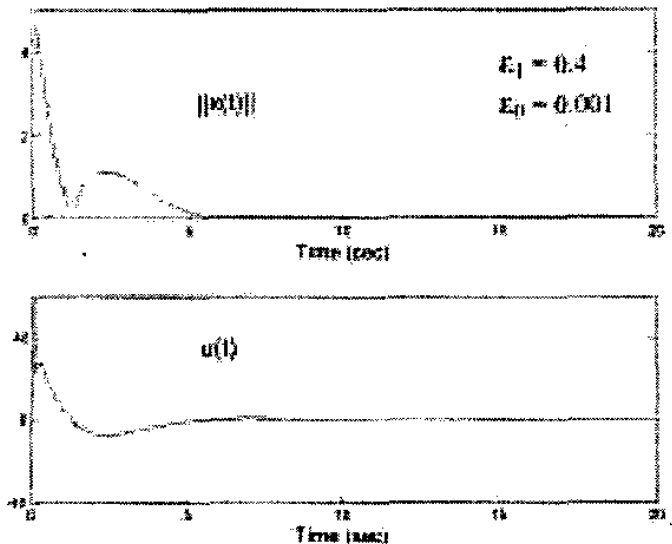

Figure 3. State-dependent boundary layer design
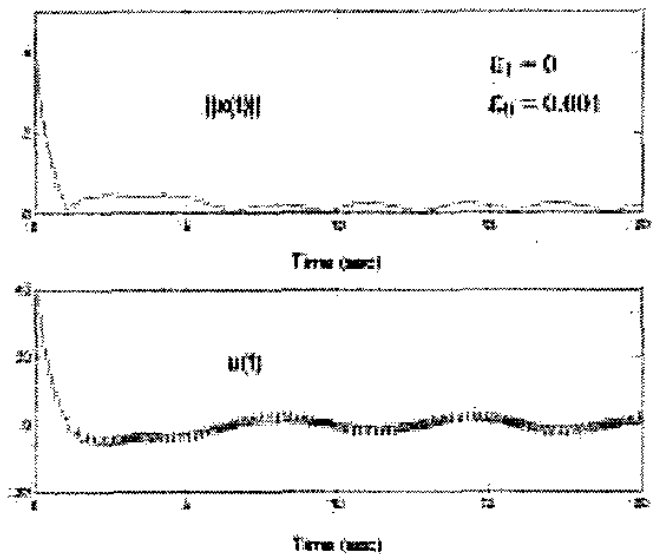

Figure 4. Constant-width boundary layer design (with disturbance )
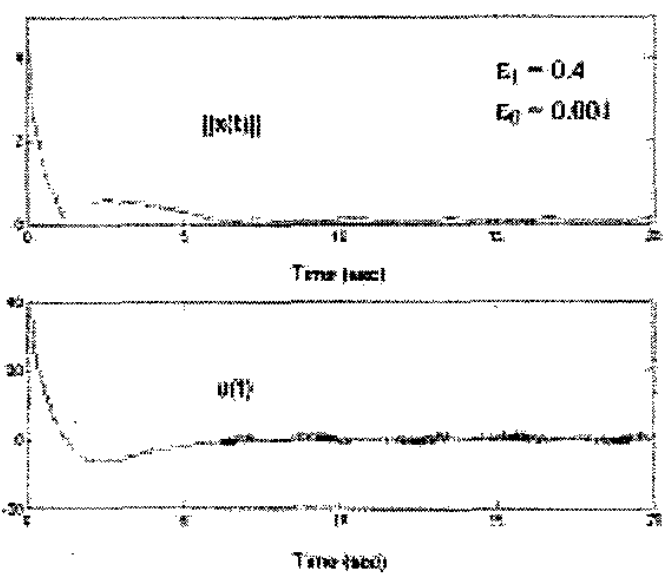

Figure 5. State-dependent boundary layer design (with disturbance) 